Journal of Animal and Veterinary Advances 11 (18): 3378-3384, 2012

ISSN: $1680-5593$

(C) Medwell Journals, 2012

\title{
The Genetic Diversity of Hainan Black Goat by Capillary Gel Electrophoresis with Microsatellite Markers in Tropical Area of China
}

\author{
H.L. Zhou, S. Guan, G. Y. Hou, D.J. Wang, T.S. Xu, \\ D.F. Wang, L.G. Shi, G. Rong and M. Li \\ Institute of Tropical Grassland and Livestock Research, \\ Chinese Academy of Tropical Agricultural Sciences, 571737 Danzhou, Hainan, China
}

\begin{abstract}
The genetic diversity and relationships of Hainan Black goat breed among 8 populations were studied using 14 microsatellite markers. In the 14 microsatellite loci, the number of alleles per locus ranged from 15 (BMS1248) to 44 (BMS2258) and the Ho values ranged from 0.003 (BM6404) to 0.923 (BM6444) among the 8 groups, the mean Polymorphic Information Content (PIC) of all the primers detected for all groups showed high values (0.892). For all the goat groups, the mean number of alleles ranged from 9.577 (Baisha) to 16.571 (Saya), the average observed heterozygotes, expected heterozygosity, Polymorphism Information Content (PIC), allelic richness and effective number of alleles was $0.402,0.866,0.838,7.625$ and 12.425 , respectively. Most of the 14 loci were in Hardy-Weinberg equilibrium ( $(\mathrm{p}>0.05)$ except locus BMS2258 $(\mathrm{p}<0.0001)$ and all the 8 populations were in Hardy-Weinberg equilibrium $(\mathrm{p}>0.05)$ too. The percentage of variation among the 8 goat groups only amounted to $5.11 \%$, the remaining existed among individuals within populations $(58.12 \%)$ and within individuals $(36.77 \%)$. The genetic differentiation pattern and genetic relationships among the 8 populations displayed some degree of consistency with their geographical locations expect Sanya and Linshui regions and suggested that exist a certain inbreeding between SY and CM. The Fis indicated some degree inbreeding existed in Changjiang goat group and there was no significant among the 8 populations. This study will help to interpret the genetic characters of Hainan Black goat and benefit to the future conservation programs.
\end{abstract}

Key words: Genetic diversity, Hainan Black goat, capillary gel electrophoresis, microsatellite markers, heterozygotes, China

\section{INTRODUCTION}

Domesticated goats (Capra hircus) play a very important role in people's life because of their unique meat, milk, fibre and skin. In particular, the lamb placenta extracted from the embryos of goats cause more attention in recent years because of its high nutritional and medicinal value, especially which is a very precious beauty products for woman. Also, there was evidence that some goat breeds are used as source of power for farm work in some communities (Morand-Fehr, 1993). But the supply of all these resource is poor and deficiency according to the traditional breeding system from the farmers. So, it was necessary to breeding all those goat breeds which had good characteristic and high product by scale and standard feeding method. First of all, it is urgent for studies on the description of the genetic potentials of goat from different geographical locations and continents of the world. The first step is to research the breed characteristics, phylogenetic traits and production potentials because of the conservation and exploitation of domestic animal biodiversity is a comprehensive knowledge for their existing genetic variability and the partitioning of the variability among breeds (Iamartino et al., 2005). Thus, an imminency investigation for the existing genetic variability and potentials of these local goat breeds became imperative. In China, there are about 197 million goats representing about $23 \%$ of the world's goat population of about 850 million. And China is one of the countries of the world with the richest goats comprising $>43$ native goat breeds (Qi et al., 2009). In fact, the genetic variability and relationships between these various Chinese goat breeds have not been properly investigated. Hainan Black goat is one of the mainly meat goat breed distributing in Hainan island of Chinese tropical zone. It is a well-known breed due to not only their resistance to heat and wet weather, crude feed and disease but also their delicious meat flavor with no urine smell, rich collagen and nutritious advantages which is regard as one of the four

Corresponding Author: S. Guan, Institute of Tropical Grassland and Livestock Research, Chinese Academy of Tropical Agricultural Sciences, 571737 Danzhou, Hainan, China 
famous dishes by popular consumers in Hainan province. There are about 9 million Hainan Black goats in the island and most of them belong to different smallholder farmers (Zhou et al., 2011). Up to now, few reports about the genetic diversity and structure of the Hainan Black goat. In addition, microsatellite marker is a very reliable and efficient method for evaluate the genetic variability, structure and distances among different goat breeds (Boyce et al., 1996; Barker, 1994; Qi et al., 2009). Therefore, this study is meant to investigate the genetic variability and relationships among the 8 major Hainan Black goat groups in Hainan island of China with 14 microsatellite markers. The data will be important and helpful for the genetic resources protection, pure-blood propagation, development and utilization for the breed.

\section{MATERIALS AND METHODS}

Sample collection and DNA extraction: Ear tissue samples were randomly collected from 287 genetically unrelated individuals representing the Hainan Black goat breed with 8 groups: Sanya Black goat ( $\mathrm{SY}, \mathrm{n}=48$ ), Linshui Black goat (LS, n = 47), Haikou Black goat ( $\mathrm{HK}, \mathrm{n}=30$ ), Lingao Black goat ( $\mathrm{LG}, \mathrm{n}=45$ ), Chenmai Black goat (CM, $n=26)$, Danzhou Black goat (DZ, $n=41$ ), Changjiang Black goat (CJ, $\mathrm{n}=27$ ) and Baisha Black goat $(\mathrm{BS}, \mathrm{n}=22$ ). The 8 Hainan Black goat breed were distributed in 8 different counties in Hainan island (Fig. 1). DNA was extracted following the well-established Phenolchloroform Method (Sambrook et al., 1989) and detected by $0.8 \%$ agarose gel electrophoresis. The content of DNA was estimated by ultraviolet spectrophotometer and the genome DNA was diluted to $50 \mathrm{ng} \mu \mathrm{L}^{-1}$.
Microsatellite analysis and PCR amplification: The 14 microsatellite loci were chosen from those recommended by the joint ISAG/FAO standing committee for MoDAD and most of them were also consistent with those used in European Econogene Project. Loci symbols were shown in Table 1 and the detailed information including primer sequence and annealing temperature can be obtained from the website ((http://dad.fao.org/en/refer/library/ guidelin/marker; http:/lasig.epfl.ch/projets/econogene).

PCR was carried out in a total volume of $12 \mu \mathrm{L}$ system containing $1 \mu \mathrm{L}$ of template DNA ( $\left.50 \mathrm{ng} \mu \mathrm{L}^{-1}\right), 1.2 \mu \mathrm{L}$ of $10 \times$ PCR buffer, $1 \mu \mathrm{L}$ of $20 \mathrm{mM}$ dNTPs, $0.25 \mu \mathrm{L}$ of each $10 \mu \mathrm{M}$ primer and 1 units DNA polymerase (Takara, Bio Inc.). The cycling conditions included an initial activation step at $95^{\circ} \mathrm{C}$ for $5 \mathrm{~min}, 30$ cycles of $94^{\circ} \mathrm{C}$ for $30 \mathrm{sec}$, annealing at $51.2-56.5^{\circ} \mathrm{C}(\mathrm{Tm}$ in Table 1$)$ for $30 \mathrm{sec}$ and extension at $72^{\circ} \mathrm{C}$ for $30 \mathrm{sec}$ and a final extension at $72^{\circ} \mathrm{C}$ for $10 \mathrm{~min}$. PCR was performed on a PTC-200 thermocycler (MJ Research, Inc.) and the products were detected on a $2 \%$ agarose gel.

Capillary gel electrophoresis: After PCR amplification, the PCR products were analyzed with QIAxcel DNA Screening kit provided by eGene System. The system was operated according to eGene's operation manual. Amplified DNA solution $(15 \mu \mathrm{L})$ was transferred to a $0.2 \mathrm{~mL}$ vial and was placed in the instrument sample tray. The DNA samples were automatically injected into the capillary channel and subjected to electrophoresis by selecting the AM320.mtQ Method from eGene's BioCalculator $^{\mathrm{TM}}$ Software. The quantificaion of DNA fragments was based on the integrated peak area determined by eGene's BioCalculator Software. The

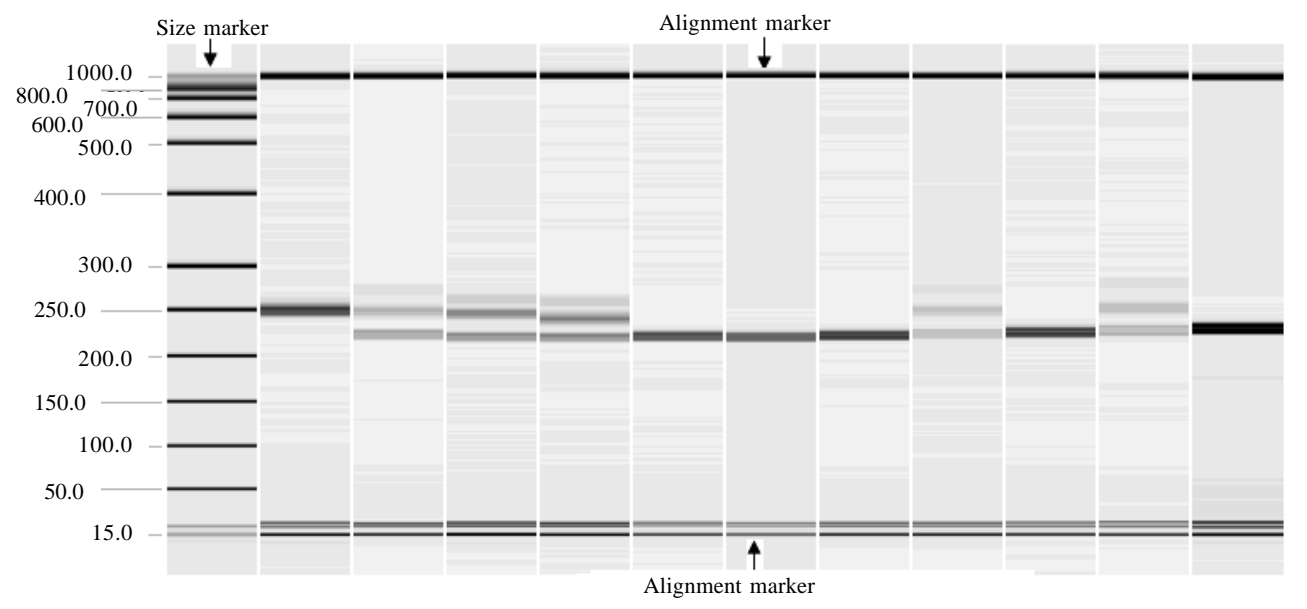

Fig. 1: The PCR fragment size of locus BM1225 analysis with QXDNA Size Marker Puc18/Haell and QX alignment marker $15 \mathrm{bp} / 1 \mathrm{~kb}$ 
J. Anim. Vet. Adv., 11 (18): 3378-3384, 2012

\begin{tabular}{|c|c|c|c|c|}
\hline Locus & Primer sequence & Chromosome distribution & Allele size & Anmealing $\left({ }^{\circ} \mathrm{C}\right)$ \\
\hline \multirow[t]{2}{*}{ BM1004 } & F:TTAAAAGTCAGAAAGGGAAGCC & 15 & $139-165$ & 55.3 \\
\hline & R:CTCGACCTCACATACTCAAAGC & & & \\
\hline \multirow[t]{2}{*}{ BM1225 } & F:TTTCTCAACAGAGGTGTCCAC & 16 & $228-298$ & 51.2 \\
\hline & R:ACCCCTATCACCATGCTCTG & & & \\
\hline \multirow[t]{2}{*}{ BM6404 } & F:TCCCTAATGTTGAATGGACTTC & 10 & $126-152$ & 52.6 \\
\hline & R:CGAAAAGAGTCAGACACCAGC & & & \\
\hline \multirow[t]{2}{*}{ INRA081 } & F:CGGCTCACGGTCTCTATCGG & 26 & $162-214$ & 53.8 \\
\hline & R:GCGAACCCAAGAATCAGACTC & & & \\
\hline \multirow[t]{2}{*}{ BP28 } & F:AGGTGACAGGTGAGAGGGC & 25 & $206-270$ & 53.5 \\
\hline & R:CCTCCACAACACCATCCTTC & & & \\
\hline \multirow[t]{2}{*}{ BM0143 } & F:ACCTGGGAAGCCTCCATATC & 6 & $100-140$ & 53.6 \\
\hline & R:CTGCAGGCAGATTCTTTATCG & & & \\
\hline \multirow[t]{2}{*}{ BMS875 } & F:TCCAGCTTGAATCCCTTCC & 19 & $112-130$ & 55.2 \\
\hline & R:AAGCAAAGGCTGGGAACAC & & & \\
\hline \multirow[t]{2}{*}{ BM1329 } & F:TTGTTTAGGCAAGTCCAAAGTC & 6 & $140-216$ & 51.5 \\
\hline & R:AACACCGCAGCTTCATCC & & & \\
\hline \multirow[t]{2}{*}{ BM1558 } & F:TGAGGAAAGCCTTGGCAG & 22 & $139-181$ & 52.6 \\
\hline & R:ACTGGGCCTAGCTCCTTCTC & & & \\
\hline \multirow[t]{2}{*}{ BM6444 } & F:CTCTGGGTACAACACTGAGTCC & 12 & $124-186$ & 55.7 \\
\hline & R:TAGAGAGTTTCCCTGTCCATCC & & & \\
\hline \multirow{2}{*}{ BMS 2258} & F:CCAGCAGAAGAGAAAGATACTGA & 7 & 149-191 & 54.9 \\
\hline & R:AGTGGTAGAACTTCCATCTCACA & & & \\
\hline \multirow[t]{2}{*}{ BM6526 } & F:CATGCCAAACAATATCCAGC & 27 & $162-218$ & 56.5 \\
\hline & R:TGAAGGTAGAGAGCAAGCAGC & & & \\
\hline \multirow[t]{2}{*}{ BMS 1248} & F:GTAATGTAGCCTTTTGTGCCG & 5 & $131-167$ & 55.8 \\
\hline & R:TCACCAACATGAGATAGTGTGC & & & \\
\hline \multirow[t]{2}{*}{ OARFCB48 } & F:AGTTAGTACAAGGATGACAAGAGGCAC & 17 & $158-196$ & 53.4 \\
\hline & R:GACTCTAGAGGATCGCAAAGAACCAG & & & \\
\hline \multirow[t]{2}{*}{ BM3413 } & F:TCCCTGGTAACCAATGAATTC & 21 & $187-225$ & 55.6 \\
\hline & R:CAATGGATTTGACCCTCCC & & & \\
\hline
\end{tabular}

BioCalculator Software calculates the DNA fragment size based on the fragment migration time in comparison to a reference QX DNA Size Marker (QXDNA Size Marker Puc18/Haell (Cat. No. 929550)) and Alignment marker (QX Alignment Marker 15 bp/1 kb (Cat. No. 929521)).

Statistical analyses: Data format conversion were calculated using the Microsatellite Toolkit (available at http://animalgenomics.ucd.ie/sdepark/ms-toolkit/). Allelic Richness (AR) was estimated using the FSTAT Program Version 2.9.3 (Goudet., 2001). F-Statistics (FIS, FIT and FST) for calculating overall genetic differentiation among populations and between pairs of populations using the variance-based method (Weir and Cockerham, 1984) of Raymond and Rousset by Genepop (Ver. 3.4) (Raymond and Rousset, 1995), allelic frequency, the exact test for Hardy-Weinberg Equilibrium (HWE) of locus-population combination and genotypic disequilibrium were estimated with Genepop (Ver. 3.4) (http://genepop.curtin.edu.au/) Software package (Raymond and Rousset, 1995).

The Number of alleles (K), Heterozygotes (Hets), Homozygotes (Homs), observed Heterozygosity (Ho) and expected Heterozygosity ( $\mathrm{He}$, Polymorphism Information Content (PIC), Null allele frequency estimate (Null freq) and accumulated exclusion probability were calculated by Cervus (Ver. 2.0)
(Marshall et al., 1998). Analysis of Molecular Variance (AMOVA) was computed using the ARLEQUIN package Version 3.11 (http://cmpg.unibe.ch/software/arlequin3/) (Schneider et al., 2000). In addition, Nei's Ds genetic distances were calculated using the Dispan program (Nei, 1978) which has a more discriminatory power for closely related groups and it is the most appropriate genetic distance measure for loci with different rate of neutral mutation (Nei et al., 1983). The neighbor-joining algorithm was used to construct a phylogenetic tree with the Dispan. Tree robustness was evaluated by bootstrapping over loci (1000 replicates) (Nei, 1978).

\section{RESULTS AND DISCUSSION}

Genetic variability: A total of 287 samples at 14 microsatellite markers were detected in the 8 Hainan Black goat groups. The number of alleles per locus ranged from 15 (BMS1248) to 44 (BMS2258) among the 8 groups. The number of heterozygotes was detected at BM6444 was the highest with 265 samples and the lowest heterozygotes was detected at BM6404 with only one sample. The Ho values ranged from 0.003 (BM6404) to 0.923 (BM6444). The Polymorphic Information Content (PIC) ranged from 0.831 (BMS1248) and 0.935 (BMS2258). The allelic richness were between 7.956(BMS2258) and 14.154 
Table 2: The average genetic polymorphism parameters of the 14 loci for the 8 goat groups

\begin{tabular}{lcccccccccc}
\hline Locus & $\mathrm{K}$ & $\mathrm{N}$ & Hets & Homs & $\mathrm{H}(\mathrm{o})$ & $\mathrm{H}(\mathrm{e})$ & $\mathrm{PIC}$ & \multicolumn{1}{c}{ AR } & Ne & Null frequency \\
BP28 & 16.00 & 287 & 38 & 249 & 0.132 & 0.889 & 0.877 & 10.131 & 9.001 & 0.7411 \\
BM1558 & 37.00 & 287 & 166 & 121 & 0.578 & 0.934 & 0.929 & 13.548 & 15.152 & 0.2363 \\
BMS2258 & 44.00 & 287 & 139 & 148 & 0.484 & 0.940 & 0.935 & 14.154 & 16.672 & 0.3194 \\
OARFCB & 26.00 & 287 & 118 & 169 & 0.411 & 0.906 & 0.896 & 10.377 & 10.643 & 0.3729 \\
BM6444 & 40.00 & 287 & 265 & 22 & 0.923 & 0.938 & 0.934 & 14.127 & 16.131 & 0.0045 \\
INRA081 & 26.00 & 287 & 131 & 156 & 0.456 & 0.931 & 0.925 & 12.907 & 14.495 & 0.3430 \\
BM0143 & 19.00 & 287 & 132 & 155 & 0.460 & 0.907 & 0.898 & 12.012 & 10.756 & 0.3248 \\
BM1329 & 20.00 & 287 & 95 & 192 & 0.331 & 0.908 & 0.899 & 11.954 & 10.874 & 0.4669 \\
BM3413 & 23.00 & 287 & 7 & 280 & 0.024 & 0.906 & 0.896 & 9.547 & 10.647 & 0.9476 \\
BMS1004 & 24.00 & 287 & 89 & 198 & 0.310 & 0.890 & 0.879 & 10.908 & 9.095 & 0.4855 \\
BMS1248 & 15.00 & 287 & 13 & 274 & 0.045 & 0.849 & 0.831 & 8.752 & 6.628 & 0.8993 \\
BM6526 & 33.00 & 287 & 71 & 216 & 0.247 & 0.931 & 0.926 & 12.233 & 14.496 & 0.5819 \\
BM6404 & 17.00 & 287 & 1 & 286 & 0.003 & 0.855 & 0.837 & 7.956 & 6.901 & 0.9919 \\
BM1225 & 43.00 & 287 & 98 & 189 & 0.341 & 0.952 & 0.948 & 12.882 & 20.833 & 0.4738 \\
Overall & 27.36 & 287 & - & - & 0.336 & 0.357 & 0.892 & 11.535 & 12.310 & 0.5165 \\
\hline
\end{tabular}

Table 3: The average genetic polymorphism parameters for the 8 goat groups

\begin{tabular}{lcccccccc}
\hline Parameters & $\mathrm{N}$ & $\mathrm{K}$ & $\mathrm{Ho}$ & $\mathrm{He}$ & $\mathrm{PIC}$ & $\mathrm{Ne}$ & $\mathrm{AR}$ & Fis \\
\hline SY & 48 & 16.571 & 0.418 & 0.898 & 0.878 & 9.800 & 16.212 & 0.567
\end{tabular}

$\begin{array}{lllllllll}\mathrm{CM} & 47 & 12.938 & 0.423 & 0.888 & 0.859 & 8.930 & 12.571 & 0.516\end{array}$

$\begin{array}{llllllllll}\text { HK } & 30 & 13.709 & 0.407 & 0.872 & 0.845 & 7.810 & 13.286 & 0.595\end{array}$

$\begin{array}{llllllllll}\text { LG } & 45 & 11.834 & 0.416 & 0.873 & 0.851 & 7.870 & 13.357 & 0.578\end{array}$

$\begin{array}{llllllllll}\text { LS } & 26 & 11.772 & 0.420 & 0.862 & 0.837 & 7.250 & 12.214 & 0.583\end{array}$

$\begin{array}{lllllllll}\mathrm{DZ} & 41 & 12.359 & 0.349 & 0.847 & 0.819 & 6.540 & 10.614 & 0.558\end{array}$

$\begin{array}{llllllllll}\text { CJ } & 27 & 11.459 & 0.349 & 0.837 & 0.805 & 6.130 & 11.571 & 0.625\end{array}$

$\begin{array}{lllllllll}\text { BS } & 22 & 9.577 & 0.432 & 0.850 & 0.809 & 6.670 & 9.5710 & 0.539\end{array}$

\begin{tabular}{lllllllll} 
Average & 287 & 12.527 & 0.402 & 0.866 & 0.838 & 7.625 & 12.425 & 0.594 \\
\hline
\end{tabular}

(BM6404). Additionally, the locus BM1225 was detected the highest effective number of alleles and the null allele frequency estimate value ranged from 0.0045 (BM6444) to 0.9919 (BM6404), each locus genetic variability information shown in Table 2. An examination of all microsatellite markers across groups revealed that most of the 14 loci were in Hardy-Weinberg equilibrium $(p>0.05)$ except locus BMS2258 $(\mathrm{p}<0.0001)$ and all the 8 populations were in Hardy-Weinberg equilibrium ( $p>0.05)$ too. In addition as shown in Table 3 , among the 8 populations, the SY recorded the highest number of alleles (16.571), expected heterozygosity (0.898), polymorphic information content (0.878), effective number of alleles (9.80) and allelic richness (16.212), the BS had the lowest number of alleles (9.577) and allelic richness (9.571) whereas BS had the highest observed heterozygosity (0.432) and there was no significant difference in another 6 populations (Fig. 2).

Genetic differentiation: The Wright's F-statistics, HT, HS and GST for each locus were as shown in Table 4, the overall HS, HT and GST was $0.868,0.910$ and 0.046 respectively, the GST indicating that only $4.6 \%$ of the total genetic diversity was partitioned among populations and the remaining $95.4 \%$ was within populations. The mean estimates of F-statistics obtained by jackknifing over loci were: FIS $=0.5728$ (within-population inbreeding
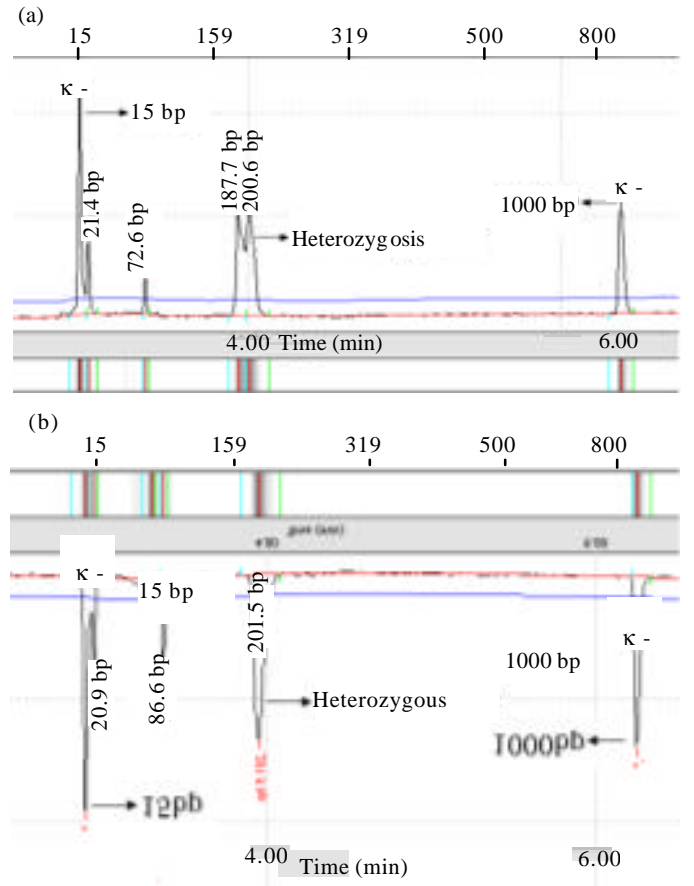

Fig. 2: The peak value of heterozygosis and homozygous for the locus OARFCB48 by Capillary gel electrophoresis analysis with QX alignment marker $15 \mathrm{bp} / 1 \mathrm{~kb}$

estimate), FIT $=0.5937$ (total inbreeding estimate), $\mathrm{FST}=0.0489$ (estimate of population differentiation). Most of the loci FIS were above zero except locus BMS2258 (-0.0540) and BM6444 (-0.0435). The FST value ranged from -0.0001 (BP28) to 0.1403 (BM1225) which was consistent with the GST ranged from -0.001 (BP28) to 0.138 (BM1 225). In addition, the FIS (0.625) of Changjiang goat group was higher than the average value $(0.594)$ which was among the 8 goat populations (Table 4). 
Table 4: Wright's F-statistics and genetic differentiation of 14 microsatellite loci for the 8 goat groups

\begin{tabular}{|c|c|c|c|c|c|c|}
\hline$\underline{\text { Locus }}$ & Fis & Fst & Fit & $\mathrm{Hs}$ & $\mathrm{Ht}$ & Gst \\
\hline BP28 & 0.8546 & -0.0001 & 0.8546 & 0.890 & 0.890 & -0.001 \\
\hline BM1558 & 0.3528 & 0.0524 & 0.3867 & 0.887 & 0.925 & 0.041 \\
\hline BMS 2258 & -0.0540 & 0.0289 & -0.0235 & 0.894 & 0.942 & 0.050 \\
\hline OARFCB & 0.5153 & 0.0776 & 0.5530 & 0.846 & 0.914 & 0.075 \\
\hline BM6444 & -0.0435 & 0.0650 & 0.0243 & 0.884 & 0.942 & 0.062 \\
\hline INRA081 & 0.4962 & 0.0361 & 0.5144 & 0.897 & 0.928 & 0.033 \\
\hline BM0143 & 0.4898 & 0.0116 & 0.4958 & 0.898 & 0.908 & 0.010 \\
\hline BM1329 & 0.6335 & 0.0151 & 0.6391 & 0.899 & 0.910 & 0.013 \\
\hline BM3413 & 0.9758 & 0.0517 & 0.9770 & 0.870 & 0.908 & 0.042 \\
\hline BMS1004 & 0.6263 & 0.0854 & 0.6582 & 0.825 & 0.890 & 0.074 \\
\hline BMS 1248 & 0.9502 & 0.0093 & 0.9507 & 0.841 & 0.850 & 0.010 \\
\hline BM6526 & 0.7239 & 0.0561 & 0.7394 & 0.886 & 0.929 & 0.047 \\
\hline BM6404 & 1.0000 & 0.0460 & 1.0000 & 0.825 & 0.857 & 0.037 \\
\hline BM1225 & 0.5936 & 0.1403 & 0.6506 & 0.815 & 0.944 & 0.138 \\
\hline Overall loci & 0.5728 & 0.0489 & 0.5937 & 0.868 & 0.910 & 0.046 \\
\hline
\end{tabular}

Table 5: Pair-wise Fst (above diagram) and genetic distance Ds (below diagram) among the 8 goat groups

\begin{tabular}{|c|c|c|c|c|c|c|c|c|}
\hline Groups & SY & $\mathrm{CM}$ & LG & $\mathrm{HK}$ & $\mathrm{DZ}$ & LS & $\mathrm{CJ}$ & BS \\
\hline SY & - & 0.03234 & 0.05354 & 0.04897 & 0.07155 & 0.04728 & 0.06018 & 0.06546 \\
\hline $\mathrm{CM}$ & 0.2463 & - & 0.06065 & 0.06414 & 0.08602 & 0.06483 & 0.08342 & 0.09161 \\
\hline LG & 0.4745 & 0.54490 & - & 0.01837 & 0.07471 & 0.01390 & 0.06578 & 0.07708 \\
\hline $\mathrm{HK}$ & 0.5148 & 0.49360 & 0.12920 & - & 0.08339 & 0.03184 & 0.07293 & 0.08161 \\
\hline DZ & 0.4853 & 0.58290 & 0.25870 & 0.09800 & - & 0.05100 & 0.03953 & 0.08460 \\
\hline LS & 0.6352 & 0.69250 & 0.71250 & 0.59150 & 0.36520 & - & 0.05496 & 0.07013 \\
\hline $\mathrm{CJ}$ & 0.5598 & 0.74100 & 0.65690 & 0.55150 & 0.46020 & 0.24400 & - & 0.03934 \\
\hline BS & 0.6067 & 0.82440 & 0.74710 & 0.66770 & 0.62390 & 0.63790 & 0.25770 & - \\
\hline
\end{tabular}

Table 6: Analysis of Molecular Variance (AMOVA) of the 8 goat groups based on 14 microsatellite DNA variations

\begin{tabular}{|c|c|c|c|c|}
\hline Source of variation & $\mathrm{df}$ & Sum of squares & Components & Percentage of variation \\
\hline Among populations & 7 & 231.051 & $0.32785 \mathrm{Va}$ & 5.11 \\
\hline Among individuals within populations & 278 & 2728.297 & $3.72781 \mathrm{Vb}$ & 58.12 \\
\hline Within individuals & 286 & 674.500 & $2.35839 \mathrm{Vc}$ & 36.77 \\
\hline Total & 571 & 3633.848 & 6.41406 & - \\
\hline
\end{tabular}

Genetic relationship and structure analysis: The AMOVA analysis revealed a low percentage of variation among populations $(5.11 \%)$, among individuals within populations $(58.12 \%)$ and within individuals $(36.77 \%)$ (Table 5). In addition, the genetic distance (Ds) (Nei, 1978) and the pair-wise genetic differentiations (FST) between populations was shown in Table 6 . The genetic distances ranged from $0.0980-0.8244$. The FST values ranged from 0.01390 for LG-LS pair to 0.09161 for BS-CM pair. The genetic distance between $\mathrm{HK}$ and $\mathrm{DZ}$ tended to be the lowest $(0.0980)$ and the widest distance was between BS and CM (0.8244). A neighbor-joining tree topology tree based on the standard genetic distance ( $\mathrm{Nei}, 1978$ ) relating the 8 goat groups studied was shown in Fig. 3 and the bootstrap values obtained in the NJ topology tree at the nodes showed the robustness of the tree. All the 8 goat groups mainly clustered two branches, HK, LG, LS, SY and CM were found in the same branch with a higher value (84\%), another branch included DZ, CJ and BS. SY and CM were grouped with a high bootstrap value (95\%). $\mathrm{HK}$ and LG clustered together with a bootstrap value $(81 \%)$. CJ and BS clustered together with a bootstrap value $(77 \%)$

According to Jandurova, heterozygosity and Polymorphic Information Content (PIC) can be used to

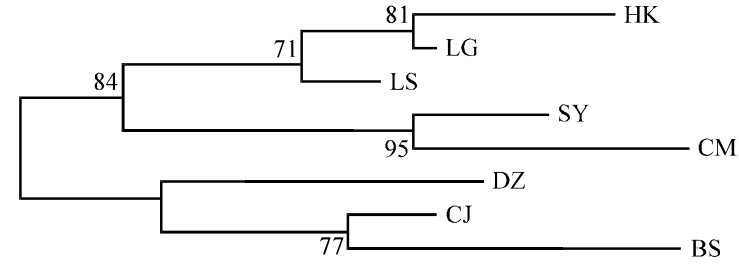

Fig. 3: Neighbor-Joining (NJ) phylogeny based on Ds genetic distances for 8 groups. Numbers at the nodes are bootstrapping values based on 1000 permutations

measure the level of genetic diversity in populations. In this study, the mean Polymorphic Information Content (PIC) of all the primers detected for all groups showed high values (0.892). It is worth to note that the locus BM6444 detected the highest observed heterozygotes (0.923), allelic richness (14.154) and lowest null allele frequency values $(0.0045)$, the result was similar to the previous studies (Arranz et al., 2001; Negrini et al., 2012), it indicates that the locus BM6444 was an excellent genetic markers with rich polymorphism information. However, the locus BM6404 detected the lowest observed heterozygotes $(0.003)$ with one samples in all populations and the highest null allele frequency (0.9919), it's 
suggested that the locus BM6404 may be was not a good genetic markers or it perhaps affected by the variety of the genetic structure of the breed. Moreover, only locus BMS2258 significantly deviated from the Hardy-Weinberg expectation ( $\mathrm{p}<0.0001)$, in all populations. On the other hand, the average polymorphic information content $(0.838)$ of Hainan Black goat breed was higher than other Chinese goat breeds (Qi et al., 2009; Guo et al., 2010; Luo et al., 2010; Fan et al., 2010) but the average observed heterozygotes $(0.402)$ was lower than those of them (Guo et al., 2010; Luo et al., 2010; Fan et al., 2010), this result suggest that Hainan Black goat breed has rich genetic diversity information and with few crossbreeding with other goat breed, it was a purebred and should be increase production. Of all these populations, SY recorded the highest genetic diversity, interestingly whereas BS had the lowest number of alleles (9.577) and allelic richness (9.571) but it had the highest observed heterozygosity (0.432). The reason why SY and BS populations had the variance genetic diversity may therefore be as follows: first, larger sample size of a population would result in more alleles (Li et al., 2002). In this study, the sample size for BS was the smallest which was only 22 , the SY population reached $48 \mathrm{sec}$ may be as for the difference genetic structure of the populations.

Although, a high genetic variation was observed, the percentage of variation among the 8 populations only amounted to $5.11 \%$ (Table 6), the gene differential coefficient $(\mathrm{Gst}=5.7 \%)$ indicated that most of total genetic diversity was due to intra-population difference and only few existed among of populations which implied these goats had relatively less genetic distinctiveness between groups. In fact, the 8 populations of Hainan Black goat was belong to a breed that distributed in different regions of Hainan island, they undergo long-term natural selection and evolution and the different ways of feeding, today leading to slightly different genetic characteristics between of them.

The relationship among populations was shown to influence the geographical location of the breeding area. In this study, the NJ topology tree showed that all the 8 goat populations mainly clustered two branches, one branch included DZ, CJ and BS, this result was consistent with the distribution of geographical location because the three counties (Danzhou, Changjiang and Baisha) located in the Eastern part of Hainan island and their geographical locations are neighboring, therefore the genetic distance and structure between the goat populations which distributing in these three areas was relatively close. Nevertheless, HK, LG, LS, SY and CM were found in the same branch with a higher value (84\%), this clustering result was not fully consistent with the

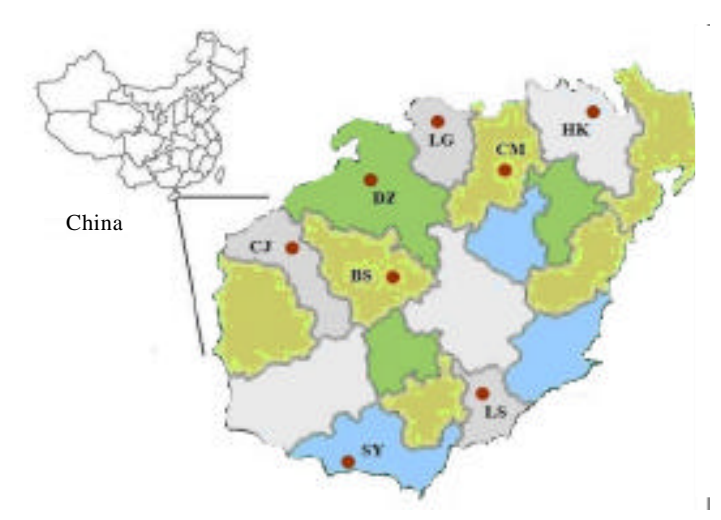

Fig. 4: Geographic distribution of 8 Hainan Black goat groups in this study

distribution of the geographical position. It is interesting that SY and CM were grouped with a high bootstrap value $(95 \%)$. Actually, according to the geographically map (Fig. 4), Sanya and Lingshui was the neighboring regions belong to the South another three counties (Haikou, Lingao and Chenmai) belong to the North but SY and CM clustered into one group while HK, LG and LS clustered together.

The results suggest that maybe exist a certain inbreeding between SY and CM, another reason was that the breeding goats of SY introduced from $\mathrm{CM}$ or $\mathrm{CM}$ breeding goats introduced from SY. However, what specific reason lead to the genetic relationship so close between these two regions populations needing further research to discuss. In addition, the Fis value of CJ (0.625) was higher than the average value of all the 8 populations, this indicate that there was a high frequency in breeding in CJ population but there was no significant in breeding between all the 8 populations.

\section{CONCLUSION}

In this study, the 14 microsatellite loci detected all the 8 Hainan Black goat populations reported high genetic diversity. Most of the 14 loci were in Hardy-Weinberg equilibrium ( $p>0.05)$ except locus BMS2258 $(p<0.0001)$ and all the 8 populations were in Hardy-Weinberg equilibrium $(p>0.05)$ too. The percentage of variation among the 8 goat groups only amounted to $5.11 \%$, the remaining existed among individuals within populations $(58.12 \%)$ and within individuals $(36.77 \%)$.

The genetic relationships among the 8 populations displayed some degree consistency with their geographical locations expect Sanya and Linshui regions and suggested that exist a certain inbreeding between SY and $\mathrm{CM}$. The Fis indicated some degree inbreeding 
existed in Changjiang goat group and there was no significant among the 8 populations. This study will help to interpret the genetic characters of Hainan Black goat and benefit to the future conservation programs.

\section{ACKNOWLEDGEMENTS}

This research was supported by National Nonprofit Institute Research Grant of CATAS-TCGRI of China (No.: 1630032012006). Researchers thank all the persons (Tie Shan Xu, Wei Ping Sun, Wan Liang Xia and Xian Zhou Huang) who helped in sampling collection. Researchers also thank associate editor of this journal and the anonymous reviewers for their constructive and professional comments.

\section{REFERENCES}

Arranz, J.J., Y. Bayon and F.S. Primitivo, 2001. Genetic variation at microsatellite loci in Spanish sheep. Small Ruminant Res., 39: 3-10.

Barker, J.S.F., 1994. A global protocol for determining genetic distance among domestic livestock breeds. Proceedings of the 5th World Congress on Genetics Applied to Livestock Production, Volume 21, August 7-12, 1994, University of Guelph, Ontario, pp: $501-508$.

Boyce, W.M., P.W. Hedrick, N.E. Muggli-Cockett, S. Kalinowski, M.C. Penedo and R.R. Ramey, 1996. Genetic variation of major histocompatibilty complex and microsatellite loci: A comparison in Bighorn sheep. Genetics, 145: 421-433.

Fan, R., Z.J. Wang, C.S. Liu, J. Luo and G.X. Zhang et al., 2010. Genetic diversity and population structure in nine chinese indigenous goat breeds. Chin. Anim. Husbandry Vet., 37: 95-99.

Goudet, J., 2001. FSTAT, a program to estimate and test gene diversities and fixation indices (version 2.9.3). http://www.unil.ch/izea/softwares/fstat.html.

Guo, D., D. Han, C. Y. Wang, X. Zheng, Y. Gao, J. Wang and H.Z. Jiang, 2010. Genetic diversity of seven microsatellite markers in liaoning cashmere goat. Chin. Anim. Husbandry Vet., 37: 99-103.

Iamartino, D., A. Bruzzone, A. Lanza, M. Blasib and F. Pillaa, 2005. Genetic diversity of Southern Italian goat populations assessed bymicrosatellite markers. Small Rumin. Res. 57: 249-255.
Li, M.H., S.H. Zhao, C. Bian, H.S. Wang and H. Wei et al., 2002. Genetic relationships among twelve Chinese indigenous goat populations based on microsatellite analysis. Genet. Selection Evol., 34: 729-744.

Luo, H.D., Y.Y. Mao, L. Zhang, H.H. Guo and X. Li et al., 2010. Polymorphism research of microsatellite loci in six local breeds of sheep and goat in Shanxi province. Acta Ecol. Anim. Domastici, 31: 17-24.

Marshall, T.C., J. Slate, L. Kruuk and J.M. Pemberton, 1998. Statistical confidence for likelihood-based paternity inference in natural populations. Mol. Ecol., 7: 639-655.

Morand-Fehr, P., 1993. Etat de la recherche caprine, notamment sur le lait de chevre: Cas des pays europeens non mediterraneens. Lait, 73: 455-464.

Negrini, R., M. D'Andrea, P. Crepaldi, L. Colli, L. Nicoloso et al., 2012. Effect of microsatellite outliers on the genetic structure of eight Italian goat breeds. Small Ruminant Res., 103: 99-107.

Nei, M., 1978. Estimation of average heterozygosity and genetic distance from a small number of individuals. Genetics, 89: 583-590.

Nei, M., F. Tajima and Y. Tateno, 1983. Accuracy of estimated phylogenetic trees from molecular data. II. Gene frequency data. J. Mol. Evol., 19: 153-170.

Qi, Y., J. Luo, X.F. Han, Y.Z. Zhu, C. Chen, J.X. Liu and H.J. Sheng, 2009. Genetic diversity and relationships of 10 Chinese goat breeds in the Middle and Western China. Small Ruminant Res., 82: 88-93.

Raymond, M. and F. Rousset, 1995. Genepop (version 1.2): Population genetics software for exact tests and ecumenicism. J. Hered., 86: 248-249.

Sambrook, J., E.F. Fritsh and T. Maniatis, 1989. Molecular Cloning: A Laboratory Manual. Cold Spring Harbor Laboratory, New York, USA.

Schneider, S., D. Roessli and L. Excoffier, 2000. ARLEQUIN: A Software for Population Genetic Data Analysis. Version 2.000, Genetics and Biometry Laboratory, University of Geneva, Switzerland.

Weir, B.S. and C.C. Cockerham, 1984. Estimating F-statistics for the analysis of population structure. Evolution, 38: 1358-1370.

Zhou, H., M. Li, X. Zi, T. Xu and G. Hou, 2011. Nutritive value of several tropical legume shrubs in Hainan sprovince of China. J. Anim. Vet. Adv., 10: 1640-1648. 